\section{Severe pulmonary air-leakage associated with bi-level positive airway pressure}

\author{
Francisco Miguel González Valverde, \\ María Jesús Gómez Ramos, ${ }^{2}$ \\ Miguel Ruiz Marín, ${ }^{1}$ \\ Marcelino Méndez Martínez ${ }^{1}$ \\ Departments of 'Surgery and \\ ${ }^{2}$ Intensive Care Unit, Reina Sofia \\ University General Hospital, \\ University of Murcia, Spain
}

\begin{abstract}
The discovery of a severe pulmonary airleakage (PAL) associated with bi-level positive airway pressure (BiPAP) is a truly strange clinical finding, even for a very experienced specialist in pulmonary medicine. We present a woman who developed pneumomediastinum, pneumoperitoneum, retropneumoperitoneum and subcutaneous emphysema while being treated with BiPAP for hypoxic respiratory failure associated with pneumonia. The pulmonary barotrauma completely resolved after discontinuation of BiPAP. PAL following spontaneous BiPAP is benign and self-limited.
\end{abstract}

\section{Introduction}

Spontaneous bi-level positive airway pressure (BiPAP; Respironics Inc.; Murrysville, PA, USA) is an effective method of non-invasive respiratory assistance.This process prevents the need or delay for endotracheal intubation and assisted mechanical ventilation but unfortunately, also can suffer from the same disadvantage: a heightened risk of barotraumas. ${ }^{1,2}$

Reported complications of BiPAP use include nasal dryness and congestion, claustrophobia, facial skin abrasions, air-leaks and conjunctivitis. However, very few cases are reported as having an association with pulmonary air-leakage (PAL), such as pneumomediastinum, pneumoperitoneum and subcutaneous emphysema. ${ }^{3,4}$

We herein present a patient treated with facemask BiPAP who developed a severe pulmonary barotrauma. Possible mechanisms involved in this unusual complication are reviewed.

\section{Case Report}

A 74-year-old female with a history of poorly controlled hypertensive cardiopathy, diabetes, hypothyroidism and chronic obstructive pulmonary disease was admitted to our Emergency Department (ED) because of pneumonia and hypoxic respiratory failure. The patient was alert but mildly dyspneic; her respiratory rate was 26 breaths/min and auscultation revealed bilateral coarse breath sounds. Her temperature was $37.7^{\circ} \mathrm{C}$, pulse rate was 110beats/min and blood pressure was 170/100 mmHg. Blood analysis demonstrated leukocytosis $\left(14800 / \mathrm{mm}^{3}\right)$ and anemia (hemoglobin $10.5 \mathrm{~g} / \mathrm{dL})$. The arterial blood gas values, with $40 \% \mathrm{FlO}_{2}$, disclosed a $\mathrm{PaO}_{2}$ of $87 \mathrm{mmHg}$, a $\mathrm{PaCO}_{2}$ of $63 \mathrm{mmHg}$, and a $\mathrm{pH}$ value of 7.33 . Chest $\mathrm{x}$-ray showed bilateral interstitial infiltrates and she was treated with intravenous ceftriaxone, levofloxacin and methylprednisolone.

BiPAP mask was applied with 18 respirations/min, an inspiratory pressure of $18 \mathrm{~cm}$ $\mathrm{H}_{2} \mathrm{O}$, an expiratory pressure of $9 \mathrm{~cm} \mathrm{H}_{2} \mathrm{O}$, and an oxygen flow rate of $12 \mathrm{~L} / \mathrm{min}$.

During the next $24 \mathrm{~h}$, she became less dyspneic and had adequate oxygenation $\left(\mathrm{PO}_{2}: 90\right.$ $\mathrm{mmHg} ; \mathrm{PCO}_{2}: 55 \mathrm{mmHg}, \mathrm{pH}: 7.38$ ) so a gradual decrease in ventilatory pressure was executed under monitoring of blood gases.

The current patient's main problem was not oxygen saturation but $\mathrm{CO}_{2}$ retention that resulted in respiratory acidosis. It is not advisable, in a patient with chronic respiratory insufficiency, to achieve an abrupt change in the level of $\mathrm{PO}_{2}$ and $\mathrm{PCO}_{2}$, and also small changes have a great clinical impact with decreased respiratory rate. The main clinical effect was the associated symptoms improvement and the normalization of $\mathrm{pH}$. A chronic respiratory patient hardly reaches higher $\mathrm{O}_{2}$ sat. 93-95.

She continued to tolerate nighttime BiPAP and was transferred to the ward after a $36 \mathrm{~h}$ stay in the ED. Four hours later, she developed increased fatigue with upper thoracic, neck and facial crepitus. Chest radiograph revealed a thin radiolucent area outlining the right mediastinum, suggesting possible pneumomediastinum. A contrast computed tomography (CT) scan showed the presence of a pneumomediastinum, pneumoperitoneum, retropneumoperitoneum and subcutaneous emphysema (Figures 1 and 2). The patient appeared dyspneic but remained apyretic and hemodynamically stable. Physical examination of the abdomen and blood tests at that time were unremarkable.

BiPAP was promptly discontinued and the patient was then treated with $100 \%$ nonrebreathing facemask. Serial chest radiographs
Correspondence: Miguel González Valverde, C/ Victorio $n^{0} 3,2^{\circ} \mathrm{C}, 30.003$ Murcia, Spain. Tel. +34.868.915.768 - Fax: +34.968 .359 .653 . E-mail:migova@terra.es

Key words: barotrauma, bi-level positive airway pressure, pneumomediastinum, pneumoperitoneum, retropneumoperitoneum and subcutaneous emphysema.

Conflicts of interests: the authors have no potential conflicts of interests.

Contributions: FMGV, conception and design, data acquisition, manuscript drafting; MJGR, data analysis and interpretation; administrative, technical and material support; MRM, supervision, data analysis and interpretation; MMM, administrative, technical and material support, supervision, manuscript critical revision for important intellectual content.

Received for publication: 13 October 2011.

Revision received: 15 January 2012.

Accepted for publication: 17 January 2012.

This work is licensed under a Creative Commons Attribution NonCommercial 3.0 License (CC BYNC 3.0).

(C) Copyright F.M. González Valverde, et al., 2012 Licensee PAGEPress, Italy

Chest Disease Reports 2012; 2:e1

doi:10.4081/cdr.2012.e1

and a new CT scan over the following 72-h period revealed complete resolution of the anomalous air while bilateral infiltrates improved.

Over the next 9 days she recovered and was discharged without sequel.

\section{Discussion}

Pneumoperitoneum, retropneumoperitoneum and subcutaneous emphysema refers to the presence of lungs air outside the thorax, a condition that is much less common than either pneumothorax or pneumomediastinum. Pneumomediastinum results from pulmonary air dissecting at the reflection of the visceral to parietal pleura near the ostia of the pulmonary veins. This occurs more frequently in infants than in adults. This is probably due to the stronger adhesions between the pleural layers in the adult, precluding communication between the pulmonary space and the mediastinum. $^{3}$

The reported patient had not undergone any attempt of venous catheterism and did not have previous surgery; hence the alteration of the normal mediastinal structures cannot have allowed air to enter directly into the other 


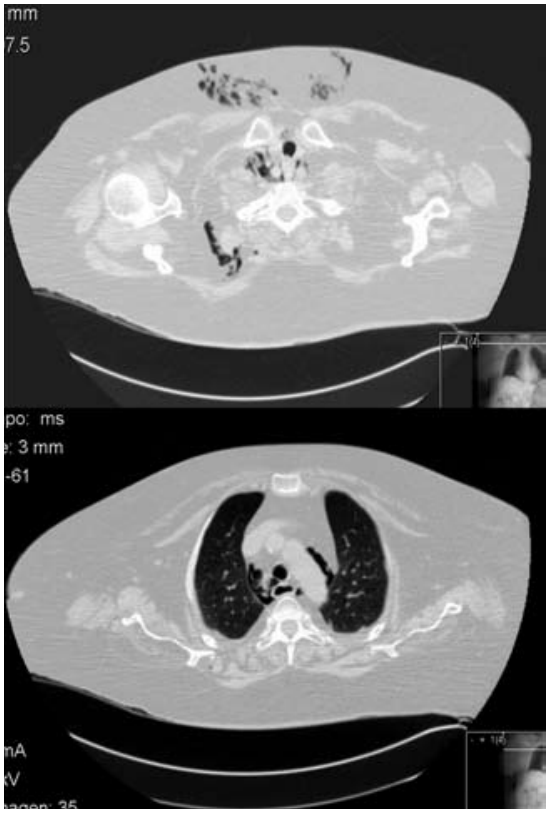

Figure 1. Chest computed tomography. Lung windows showing pneumomediastinum and subcutaneous emphysema over the anterior chest. Radiographically, pneumomediastinum and pneumothorax frequently are confused since they can occur concomitantly. In this case, pneumothorax was refused.

spaces from the mediastinum. She had not any clinical reason to be predisposed to this event. The anatomic route by which pneumomediastinum and pneumothoraces results in peritoneal air was described by Maunder et al. ${ }^{5}$ The soft tissue compartment of the neck, thorax and abdomen contains four regions defined as the subcutaneous tissue, prevertebral tissue, visceral space and previsceral space. The visceral space inverts the trachea and esophagus and continues with these structures into the mediastinum and bronchovascular sheaths. It follows the esophagus though the diaphragmatic hiatus into the retroperitoneal and peritoneal soft tissue space. Thus, there is conti-

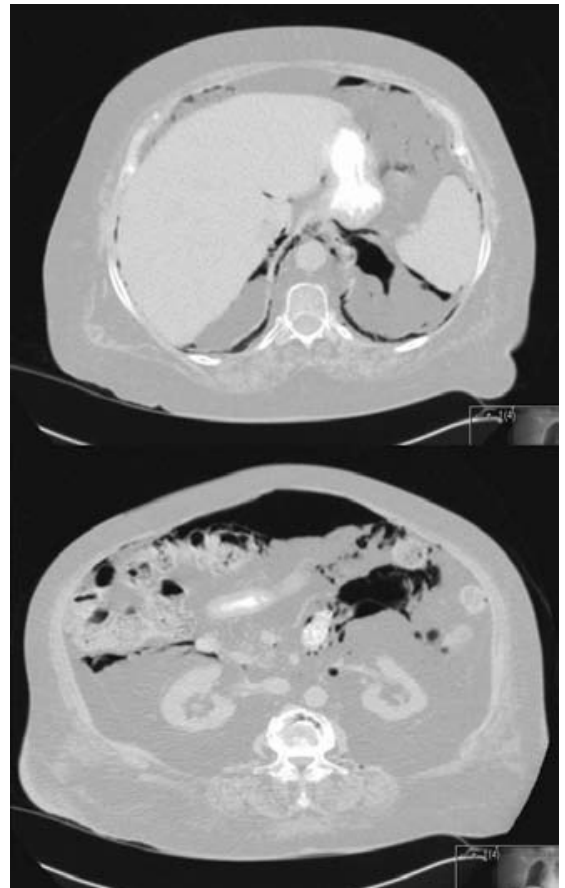

Figure 2. Contrast enhanced computed tomography scan. Abdomen showing pneumoperitoneum and retropneumoperitoneum. Free esophagogastric perforation was refused.

nuity along the neck, thorax and abdomen. Air arising in any one of these regions could reach another area by traveling along the fascial planes.

In mechanically ventilated patients, there is an increased incidence of PAL especially when there is an associated pulmonary parenchymal process and excessive distending airway pressure. ${ }^{3,4}$ The underlying chronic obstructive pulmonary disease by itself could be responsible for PAL during an acute exacerbation. So, our patient probably developed alveolar rupture and BiPAP contributed to increasing the pressure gradient between alveoli and the bronchovascular sheath leading to pneumomedi- astinum and pneumoperitoneum. Furthermore, air resolved completely once the BiPAP was discontinued.

Management of pneumoperitoneum is conservative, similar to that of pneumomediastinum. Surgical intervention would be appropriate in patients with concomitant hemodynamic compromise. Also, these patients need to be observed closely since they are at high risk for other barotrauma complications. ${ }^{4}$ In this case, the PAL resolved soon after the discontinuation of BiPAP and initiation of treatment with high-inspired oxygen.

\section{Conclusions}

PAL following spontaneous BiPAP is benign, self-limited and needs no surgical or radiological intervention.

\section{References}

1. Mehta S, Hill NS. Noninvasive ventilation. Am J Respir Crit Care Med 2001;163:54077.

2. Clinical indications for noninvasive positive pressure ventilation in chronic respiratory failure due to restrictive lung disease, COPD, and nocturnal hypoventilation: a consensus conference report. Chest 1999;116:521-34.

3. Marcy TW. Barotrauma: detection, recognition, and management. Chest 1993;104: 578-84.

4. McEachern RC, Patel RG. Pneumopericardium associated with face-mask continuous positive airway pressure. Chest 1997;112:1441-3.

5. Maunder RJ, Pierson DJ, Hudson LD. Subcutaneous and mediastinal emphysema. Pathophysiology, diagnosis, and management. Arch Intern Med 1984;144:144753 . 